\section{(d) \\ CrossMark}

\title{
The contribution of infection and the respiratory microbiome in acute exacerbations of idiopathic pulmonary fibrosis
}

\author{
Rachele Invernizzi ${ }^{1}$ and Philip L. Molyneaux ${ }^{1,2}$ \\ Affiliations: ${ }^{1}$ Fibrosis Research Group, National Heart and Lung Institute, Imperial College, London, UK. ${ }^{2}$ Dept \\ of Respiratory Medicine, Interstitial Lung Disease Unit, Royal Brompton Hospital, London, UK. \\ Correspondence: Philip L. Molyneaux, Fibrosis Research Group, National Heart and Lung Institute, Imperial \\ College, London, SW7 2AZ, UK. E-mail: p.molyneaux@imperial.ac.uk.
}

\section{@ERSpublications}

Microbial dysbosis plays a role in the pathogenesis of IPF and further changes occur during acute exacerbations despite exclusion of overt infection. http://bit.ly/2MQkfBN

Cite this article as: Invernizzi R, Molyneaux PL. The contribution of infection and the respiratory microbiome in acute exacerbations of idiopathic pulmonary fibrosis. Eur Respir Rev 2019; 28: 190045 [https://doi.org/10.1183/16000617.0045-2019].

ABSTRACT Idiopathic pulmonary fibrosis (IPF) arises in genetically susceptible individuals as a result of an aberrant wound-healing response following repetitive alveolar injury. The clinical course of the disease remains both variable and unpredictable with periods of more rapid decline, termed acute exacerbation of IPF (AE-IPF), often punctuating the disease trajectory. Exacerbations carry a significant morbidity and mortality, and their exact pathogenesis remains unclear. Given the emerging evidence that disruption and alteration in the lung microbiome plays a role in the pathogenesis and progression of IPF, this review discusses the current knowledge of the contribution of infection and the respiratory microbiome to AE-IPF.

\section{Introduction}

Although the pathogenesis of idiopathic pulmonary fibrosis (IPF) remains poorly understood the condition is thought to arise in genetically susceptible ageing individuals as a consequence of repeated microinjuries to the alveolar epithelium by environmental triggers [1]. Over time, this process results in an aberrant wound-healing response, which leads to fibrosis and causes the progressive structural destruction of the lung $[2,3]$. This suggests both environmental and host factors play a role in IPF, likely with bidirectional interaction between the two. The clinical course of the disease is both variable and unpredictable with significant heterogeneity among individual patients [4]. Whatever trajectory the disease adopts it can be punctuated by unpredictable periods of acute deterioration; episodes termed acute exacerbations of IPF (AE-IPF). These are defined as acute, clinically significant respiratory deterioration with no identifiable cause, characterised by new, widespread alveolar abnormalities [5]. The exact incidence of acute exacerbations is unclear but seems to range between 4-20\% per year with these episodes conferring a substantial morbidity and mortality (often $>50 \%$ ) [6, 7]. Known triggers for AE-IPF include surgical lung biopsy, lung resection and drugs [8-11]. Nonetheless, our current understanding of the pathophysiology of AE-IPF is limited and it remains unclear whether AE-IPF represent an accelerated

Submitted article, peer reviewed.

Received: April 242019 | Accepted after revision: June 162019

Copyright $\odot$ ERS 2019. This article is open access and distributed under the terms of the Creative Commons Attribution Non-Commercial Licence 4.0. 
phase of the underlying fibrotic condition or a response to an occult trigger. Given the growing evidence for the role of infection in the pathogenesis and progression of IPF [12, 13], in this review we seek to explore the potential contribution of infection and the microbiome in acute exacerbations.

\section{The lung microbiome: a complex and dynamic community of microbes}

The epithelial surfaces of the human respiratory tract have historically been described as sterile. More recently, novel culture-independent techniques have debunked this notion by demonstrating that the respiratory tract harbours a complex and dynamic community of microbes which play a role both in health and disease [14]. High-throughput sequencing of the 16S rRNA gene is the most commonly used approach to study bacterial communities. Groups of bacteria sharing similar gene sequences are clustered together and identified by comparison to $16 \mathrm{~S}$ rRNA reference databases. The commensal, symbiotic and pathogenic microorganisms which inhabit the lower airways are collectively defined as the "lung microbiome". In health, the primary routes of microbial immigration to the lungs include microaspiration, inhalation of air-borne bacteria and direct dispersion along mucosal surfaces [15]. Of these, microaspiration is probably the dominant mechanism, as the composition of the oral microbiome most closely resembles the lung microbiome. It is also expected to be the most relevant to IPF given the strong association with reflux [16]. Elimination is achieved through mucociliary clearance, coughing, and innate and adaptive immune defences which selectively recognise, kill and clear the microbes [17]. The quantity and composition of the microbiome is influenced by the balance between the rate of immigration and clearance. The discovery of the lung microbiome has prompted the exploration of its role in a variety of respiratory conditions, including IPF. These molecular techniques have not only enabled researchers to characterise niche-specific microbial communities in the respiratory tract of patients and healthy individuals, but have also demonstrated associations which point towards the interaction between the microbiome and the host as key in the aetiology and progression of lung disease [15, 18-20].

\section{The role of infection and the microbiome in AE-IPF}

Historically, research investigating the role of infections in the pathogenesis of IPF and as triggers of AE-IPF has focused primarily on viruses. When considering the role of infection in AE-IPF, it is important to remember these are events that, prior to the latest revised diagnostic criteria [5], specifically required the exclusion of any infective trigger [21]. Therefore, the following findings are all in cohorts of patients where overt clinical infection has been excluded.

It has been hypothesised that chronic viral infection may act as a persistent antigenic stimulus or co-factor in susceptible hosts, triggering fibrosis, and there is growing evidence both from human and animal studies to support this $[22,23]$. The human herpes virus (HHV) family has received the most attention as either an aetiologic or exacerbating agent of IPF [24, 25], but a number of other viral infections have been consistently associated with IPF and AE-IPF (table 1).

There is growing evidence, albeit associative, suggesting that subclinical or occult viral infections may play a pathogenic role in the development of AE-IPF [32]. Molecular analysis of viral particles and bronchoalveolar lavage (BAL) revealed infection in 19 out of 43 cases of AE-IPF, including 12 cases of torque teno virus (TTV) in the exacerbation cohort with no viruses detected in the stable IPF group [26]. Supporting this observation, IPF subjects who were found to be seropositive for TTV-DNA demonstrated a worse survival in an independent cohort [30]. More common respiratory viral pathogens have also been detected using molecular techniques during exacerbations, including respiratory syncytial virus and cytomegalovirus [27]. Having excluded infection clinically, a cohort of 37 IPF patients experiencing an exacerbation underwent surgical lung biopsy and over one-third exhibited evidence of viral infection based on immunohistochemistry or microarray [28]. More recently, viral sequences were profiled in nasopharyngeal swabs of 30 stable IPF and 30 AE-IPF patients which revealed higher virus-positive rates in the AE-IPF cohort (60\%) compared to stable disease (43\%) [29]. HHV was the most prominent virus in the AE-IPF group as well as influenza A [29]. As AE-IPF have been reported following influenza A vaccination, its presence suggests that cold-associated infection may potentially trigger these events [31]. Overall these observational studies highlight a potential role for viral infections, although the supporting mechanistic role for airborne viruses is insufficient to prove their causal role in AE-IPF.

While the role of viruses has been extensively explored, the long-held, but incorrect, theory of sterility of the lung outside of clinical infection means historically little work has evaluated the role of bacteria in AE-IPF. Using quantitative methods, the first study to investigate the presence of bacterial growth in the lower airways of 22 stable IPF patients confirmed the presence of known pathogens including Haemophilus, Pseudomonas and Streptococcus in 36\% of the cases [33]. More recently, molecular culture-independent techniques have been used to characterise the microbial composition in the lower 
TABLE 1 Summary of studies linking viruses with the pathogenesis, progression and acute exacerbation of idiopathic pulmonary fibrosis (IPF)

\begin{tabular}{|c|c|c|}
\hline Virus & Main conclusions & [Ref.] \\
\hline \multirow[t]{7}{*}{ HHV } & $\begin{array}{l}\text { EBV detected in serum of } 12 \text { out of } 13 \text { subjects with IPF but not in patients with } \\
\text { other forms of ILD }\end{array}$ & {$[22]$} \\
\hline & MHV-68 triggers an exaggerated fibrotic response in mice & [23] \\
\hline & $\begin{array}{l}\text { Increased incidence of EBV in BAL and lung biopsies of IPF subjects compared to } \\
\text { controls }\end{array}$ & {$[24,25]$} \\
\hline & EBV detected in BAL of two out of $43 \mathrm{AE}-I P F$ subjects & {$[26]$} \\
\hline & CMV detected in BAL of two out of $43 \mathrm{AE}-\mathrm{IPF}$ subjects & [27] \\
\hline & A total of $38 \%$ of AE-IPF subjects exhibited evidence of CMV infection & {$[28]$} \\
\hline & $\begin{array}{l}\text { HHV detected in nasopharyngeal swabs of } 15 \text { out of } 30 \text { AE-IPF subjects and four out } \\
\text { of } 30 \text { individuals with stable IPF }\end{array}$ & {$[29]$} \\
\hline \multirow[t]{3}{*}{ TTV } & TTV detected in BAL of 12 out of 43 AE-IPF subjects & [26] \\
\hline & No evidence of TTV in BAL of stable IPF subjects & [30] \\
\hline & $\begin{array}{l}\text { Increased mortality in IPF subjects with presence of TTV-DNA in serum compared } \\
\text { to IPF subjects with no TTV-DNA }\end{array}$ & \\
\hline \multirow[t]{2}{*}{ Influenza A } & $\begin{array}{l}\text { Influenza A detected in nasopharyngeal swabs of } 12 \text { out of } 30 \text { AE-IPF subjects but } \\
\text { not in individuals with stable IPF }\end{array}$ & {$[29]$} \\
\hline & A case of AE-IPF was reported following pandemic influenza A vaccination & {$[31]$} \\
\hline
\end{tabular}

airways of IPF patients. Recent studies suggest that microbial dysbiosis may be linked to disease outcome (table 2).

The Correlating Outcomes with Biochemical Markers to Estimate Time-Progression (COMET) study was the first to truly evaluate the lung microbiome in IPF patients [34]. The authors demonstrated an increased abundance of either Streptococcus or Staphylococcus was associated with a significant reduction in progression-free survival time [34]. The enrichment of these two bacteria was observed in less than half of the cohort; therefore, it remains unlikely that these organisms alone can explain the disease pathogenesis. Consistent with the COMET study, MolynEaux et al.[36] demonstrated that the lower airways of patients with IPF were not sterile and indeed were more likely to harbour potentially pathogenic Haemophilus, Neisseria and Streptococcus species than healthy controls. The authors also

TABLE 2 Summary of studies linking the respiratory microbiome with the pathogenesis, progression and acute exacerbation of idiopathic pulmonary fibrosis (IPF)

\begin{tabular}{|c|c|}
\hline Diagnosis & Main conclusions \\
\hline \multirow[t]{6}{*}{ IPF } & $\begin{array}{l}\text { Positive BAL cultures in eight out of } 22 \text { stable IPF subjects: Haemophilus influenzae } \\
\text { (n=2), Haemophilus parainfluenzae }(n=2) \text {, Moraxella catarrhalis }(n=1), \text { Pseudomonas } \\
\text { aeruginosa ( } n=1) \text {, Proteus mirabilis }(n=1) \text {, Streptococcus pneumonia }(n=1)\end{array}$ \\
\hline & $\begin{array}{l}\text { Increased abundance of Streptococcus OTU1345 and Staphylococcus OTU1348 is } \\
\text { associated with a significant reduction in progression-free survival in IPF }\end{array}$ \\
\hline & $\begin{array}{l}\text { Streptococcus pneumoniae triggers progression of pulmonary fibrosis through } \\
\text { pneumolysin in two different mouse models }\end{array}$ \\
\hline & Increased bacterial burden in IPF subjects compared with COPD and healthy controls \\
\hline & Higher bacterial burden at the time of diagnosis predicts disease progression in IPF \\
\hline & Germ-free mice protected from mortality following bleomycin exposure \\
\hline \multirow[t]{3}{*}{ AE-IPF } & Four-fold increase in bacterial burden in AE-IPF subjects compared to stable IPF \\
\hline & $\begin{array}{l}\text { Increased abundance of Campylobacter and Stenotrophomonas and decreased } \\
\text { abundance of Veillonella in AE-IPF compared to stable IPF }\end{array}$ \\
\hline & $\begin{array}{l}\text { Positive sputum cultures in nine out of } 48 \text { AE-IPF subjects: Klebsiella pneumoniae } \\
\text { (n=2), Mycobacterium tuberculosis }(n=4), \text { Pseudomonas aeruginosa }(n=1), \text { Loffi } \\
\text { Acinetobacter }(n=1) \text {, other }(n=1)\end{array}$ \\
\hline
\end{tabular}

AE-IPF: acute exacerbation of IPF; BAL: bronchoalveolar lavage. 
identified the presence of a higher overall bacterial burden in IPF, which was associated with a reduced progression-free survival time in subjects with IPF. Having characterised the microbiome in stable IPF, the same authors then set out to examine for potential changes during acute exacerbations. Using a cohort of matched stable and exacerbation patients who underwent bronchoscopy, the authors were able to demonstrate a further increase in bacterial burden during an exacerbation, despite the presence of negative clinical cultures [38]. Subjects experiencing an AE-IPF exhibited a marked change in the respiratory microbiome with an increase in Campylobacter and Stenotrophomonas species compared to stable disease. Both of these organisms are potential respiratory pathogens but the presence of Campylobacter also leads the authors to suggest their findings support the role of reflux and aspiration with AE-IPF. One weakness of the study was that there were only two paired samples from the same individuals when stable and experiencing an exacerbation. The stark changes in these two pairs of samples is intriguing, especially as in one individual there was a marked outgrowth of streptococcal species from the baseline microbiota which then dominated the exacerbation state. This result is concordant with findings from two distinct mouse models, where infection with Streptococcus pneumoniae induced exacerbation of established lung fibrosis [35].

More recently, WENG et al. [29] have employed molecular techniques to examine sputum cultures of 170 patients experiencing an exacerbation. They detected 38 different bacterial strains with gram-negative bacteria accounting for $89 \%$ of pathogens: Klebsiella pneumonia (26\%), Mycobacterium tuberculosis (21\%), and Acinetobacter baumannii (10\%) dominated the sputum samples [29]. Together these studies demonstrate that alterations in the respiratory microbiome occur during acute exacerbations but they do not provide any functional or mechanistic insights. In a recent study, O'DwYER et al. [37] tried to overcome this issue in an attempt to move from correlation to causation. A germ-free mouse model of fibrosis was used to explore the role of bacteria on lung inflammation and fibrinogenesis. Following bleomycin exposure, germ-free mice were protected from mortality, even though they exhibited similar severity of pulmonary fibrosis when compared to conventional mice [37]. The authors postulate that this may reflect the clinical observation that subjects with IPF not only die from progressive fibrosis but also from distinct inflammatory causes of acute-on-chronic respiratory failure including respiratory infections and AE-IPF. While there are several limitations of animal models in IPF, collectively, this study shows how preclinical germ-free models are a useful tool to investigate potential mechanisms of host-microbiota interactions and provides the first causal evidence that the microbiome participates in the pathogenesis and mortality of fibrotic lung disease.

\section{Conclusions and future directions}

The role of the respiratory microbiome in the pathogenesis and progression of IPF is just beginning to be elucidated. Clinically, acute exacerbations are important events in the natural course of IPF which carry a significant morbidity and mortality. While there is a paucity of information regarding the aetiology, pathophysiology and clinical management of acute exacerbations, an increasing number of findings indicate that infection, both viral and bacterial might be involved. Recent advances in sequencing technologies have allowed the use of molecular microbial technologies to characterise the respiratory microbiota in these patients demonstrating a clear change in the respiratory microbiome, as well as an increased bacterial burden in BAL compared to stable disease. However, the microbiome has not been fully characterised and further research should also investigate organisms other than bacteria and viruses, including fungi. It is unclear whether a higher bacterial load and an altered microbiome during an acute exacerbation reflect an active infection, increased aspiration or occur as a result of widespread diffuse alveolar damage. As sequencing DNA from BAL provides a snapshot in time of the microbial diversity, future research should incorporate longitudinal sampling before, during and after an AE-IPF. Furthermore, there is a pressing need to move from correlation to causation [39]. In order to do so, functional studies examining host-microbiome interactions as well as better models of AE-IPF are needed, given existing models fail to fully recapitulate physiologic findings in IPF. Current consensus is that all patients experiencing an exacerbation should receive broad spectrum empirical antibiotics even if no overt infection can be identified [40]. While this is likely driven by the use of immunosuppressive agents, the molecular microbial data may support this empiric therapy. Indeed, there is already some evidence that azithromycin may be beneficial in AE-IPF [41]. Ultimately, understanding the role of the microbiome in AE-IPF will allow its manipulation and may provide an opportunity for targeted therapeutic intervention.

Conflict of interest: R. Invernizzi has nothing to disclose. P.L. Molyneaux has, via his institution, received speaker's fees from Roche and Boehringer Ingelheim.

\section{References}

1 Maher TM, Wells AU, Laurent GJ. Idiopathic pulmonary fibrosis: multiple causes and multiple mechanisms? Eur Respir J 2007; 30: 835-839.

2 Daccord C, Maher TM. Recent advances in understanding idiopathic pulmonary fibrosis. F1000Res 2016; 5: 1046. 
(

Ley B, Collard HR, King TE Jr. Clinical course and prediction of survival in idiopathic pulmonary fibrosis. Am J Respir Crit Care Med 2011; 183: 431-440.

5 Collard HR, Ryerson CJ, Corte TJ, et al. Acute exacerbation of idiopathic pulmonary fibrosis. An International Working Group Report. Am J Respir Crit Care Med 2016; 194: 265-275.

6 Moua T, Westerly BD, Dulohery MM, et al. Patients with fibrotic interstitial lung disease hospitalized for acute respiratory worsening: a large cohort analysis. Chest 2016; 149: 1205-1214.

7 Ryerson CJ, Collard HR. Acute exacerbations complicating interstitial lung disease. Curr Opin Pulm Med 2014; 20: 436-441.

8 Kreider ME, Hansen-Flaschen J, Ahmad NN, et al. Complications of video-assisted thoracoscopic lung biopsy in patients with interstitial lung disease. Ann Thorac Surg 2007; 83: 1140-1144.

9 Bando M, Ohno S, Hosono T, et al. Risk of acute exacerbation after video-assisted thoracoscopic lung biopsy for interstitial lung disease. J Bronchology Interv Pulmonol 2009; 16: 229-235.

10 Sakamoto S, Homma S, Mun M, et al. Acute exacerbation of idiopathic interstitial pneumonia following lung surgery in 3 of 68 consecutive patients: a retrospective study. Intern Med 2011; 50: 77-85.

11 Kondoh Y, Cottin V, Brown KK. Recent lessons learned in the management of acute exacerbation of idiopathic pulmonary fibrosis. Eur Respir Rev 2017; 26: 170050.

12 Molyneaux PL, Maher TM. The role of infection in the pathogenesis of idiopathic pulmonary fibrosis. Eur Respir Rev 2013; 22: 376-381.

13 Hewitt RJ, Molyneaux PL. The respiratory microbiome in idiopathic pulmonary fibrosis. Ann Transl Med 2017; 5: 250 .

14 Dickson RP, Erb-Downward JR, Martinez FJ, et al. The microbiome and the respiratory tract. Annu Rev Physiol 2016; 78: 481-504.

15 Dickson RP, Erb-Downward JR, Huffnagle GB. The role of the bacterial microbiome in lung disease. Expert Rev Respir Med 2013; 7: 245-257.

16 Mackintosh JA, Desai SR, Adamali H, et al. In patients with idiopathic pulmonary fibrosis the presence of hiatus hernia is associated with disease progression and mortality. Eur Respir J 2019; 53: 1802412.

17 Dickson RP, Erb-Downward JR, Freeman CM, et al. Bacterial topography of the healthy human lower respiratory tract. MBio 2017; 8: e02287.

18 Hilty M, Burke C, Pedro H, et al. Disordered microbial communities in asthmatic airways. PLoS One 2010; 5 : e8578.

19 Molyneaux PL, Mallia P, Cox MJ, et al. Outgrowth of the bacterial airway microbiome after rhinovirus exacerbation of chronic obstructive pulmonary disease. Am J Respir Crit Care Med 2013; 188: 1224-1231.

20 Molyneaux PL, Willis-Owen SAG, Cox MJ, et al. Host-microbial interactions in idiopathic pulmonary fibrosis. Am J Respir Crit Care Med 2017; 195: 1640-1650.

21 Collard HR, Moore BB, Flaherty KR, et al. Acute exacerbations of idiopathic pulmonary fibrosis. Am J Respir Crit Care Med 2007; 176: 636-643.

22 Vergnon JM, Vincent M, de The G, et al. Cryptogenic fibrosing alveolitis and Epstein-Barr virus: an association? Lancet 1984; 2: 768-771.

23 Stoolman JS, Vannella KM, Coomes SM, et al. Latent infection by $\gamma$ herpesvirus stimulates profibrotic mediator release from multiple cell types. Am J Physiol Lung Cell Mol Physiol 2011; 300: L274-L285.

24 Manika K, Alexiou-Daniel S, Papakosta D, et al. Epstein-Barr virus DNA in bronchoalveolar lavage fluid from patients with idiopathic pulmonary fibrosis. Sarcoidosis Vasc Diffuse Lung Dis 2007; 24: 134-140.

25 Tang YW, Johnson JE, Browning PJ, et al. Herpesvirus DNA is consistently detected in lungs of patients with idiopathic pulmonary fibrosis. J Clin Microbiol 2003; 41: 2633-2640.

26 Wootton SC, Kim DS, Kondoh Y, et al. Viral infection in acute exacerbation of idiopathic pulmonary fibrosis. Am J Respir Crit Care Med 2011; 183: 1698-1702.

27 Ushiki A, Yamazaki Y, Hama M, et al. Viral infections in patients with an acute exacerbation of idiopathic interstitial pneumonia. Respir Investig 2014; 52: 65-70.

28 Santos GC, Parra ER, Stegun FW, et al. Immunohistochemical detection of virus through its nuclear cytopathic effect in idiopathic interstitial pneumonia other than acute exacerbation. Braz J Med Biol Res 2013; 46: 985-992.

29 Weng D, Chen XQ, Qiu H, et al. The role of infection in acute exacerbation of idiopathic pulmonary fibrosis. Mediators Inflamm 2019; 2019: 5160694.

30 Bando M, Ohno S, Oshikawa K, et al. Infection of TT virus in patients with idiopathic pulmonary fibrosis. Respir Med 2001; 95: 935-942.

31 Umeda Y, Morikawa M, Anzai M, et al. Acute exacerbation of idiopathic pulmonary fibrosis after pandemic influenza A (H1N1) vaccination. Intern Med 2010; 49: 2333-2336.

32 Vannella KM, Moore BB. Viruses as co-factors for the initiation or exacerbation of lung fibrosis. Fibrogenesis Tissue Repair 2008; 1: 2.

33 Richter AG, Stockley RA, Harper L, et al. Pulmonary infection in Wegener granulomatosis and idiopathic pulmonary fibrosis. Thorax 2009; 64: 692-697.

34 Han MK, Zhou Y, Murray S, et al. Lung microbiome and disease progression in idiopathic pulmonary fibrosis: an analysis of the COMET study. Lancet Respir Med 2014; 2: 548-556.

35 Knippenberg S, Ueberberg B, Maus R, et al. Streptococcus pneumoniae triggers progression of pulmonary fibrosis through pneumolysin. Thorax 2015; 70: 636-646.

36 Molyneaux PL, Cox MJ, Willis-Owen SA, et al. The role of bacteria in the pathogenesis and progression of idiopathic pulmonary fibrosis. Am J Respir Crit Care Med 2014; 190: 906-913.

37 O'Dwyer DN, Ashley SL, Gurczynski SJ, et al. Lung microbiota contribute to pulmonary inflammation and disease progression in pulmonary fibrosis. Am J Respir Crit Care Med 2019; 199: 1127-1138.

38 Molyneaux PL, Cox MJ, Wells AU, et al. Changes in the respiratory microbiome during acute exacerbations of idiopathic pulmonary fibrosis. Respir Res 2017; 18: 29.

39 Segal LN, Molyneaux PL. The challenging road of moving from association to causation for microbiome research in IPF. Am J Respir Crit Care Med 2019; 199: 1054-1056. 
40 Maher TM, Whyte MK, Hoyles RK, et al. Development of a consensus statement for the definition, diagnosis, and treatment of acute exacerbations of idiopathic pulmonary fibrosis using the Delphi technique. Adv Ther 2015; 32: 929-943.

41 Macaluso C, Furcada JM, Alzaher O, et al. The potential impact of azithromycin in idiopathic pulmonary fibrosis. Eur Respir J 2018; 53: 1800628. 